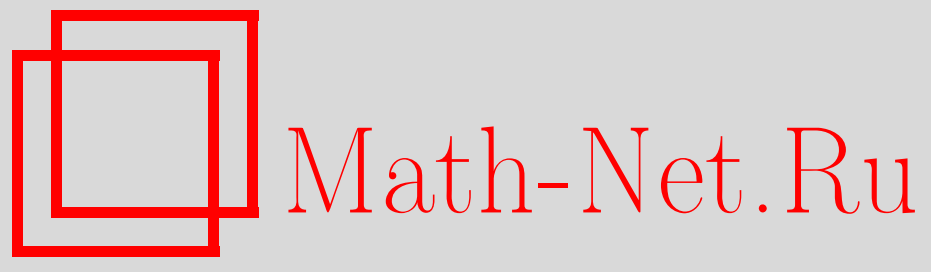

И. В. Подвигин, Об экспоненциальной скорости сходимости в эргодической теореме Биркгофа, Матем. заметки, 2014, том 95, выпуск 4, 638-640

DOI: https://doi.org/10.4213/mzm10430

Использование Общероссийского математического портала Math-Net.Ru подразумевает, что вы прочитали и согласны с пользовательским соглашением http://www . mathnet.ru/rus/agreement

Параметры загрузки:

IP: 35.174 .16 .151

26 апреля 2023 г., 17:39:09






\section{Об экспоненциальной скорости сходимости в эргодической теореме Биркгофа}

\section{И. В. Подвигин}

Пусть $(\Omega, \lambda)$ - пространство с вероятностной мерой, $T$ - его эндоморфизм. Для $f \in$ $L_{1}(\Omega), \omega \in \Omega, n \in \mathbb{N}$ обозначим

$$
A_{n} f(\omega)=\frac{1}{n} \sum_{k=0}^{n-1} f\left(T^{k} \omega\right)
$$

Эргодическая теорема Биркгофа утверждает, что для таких средних существует $\lambda$-п.в. предел $f^{*}=\lim _{n \rightarrow \infty} A_{n} f$; в случае эргодичности эндоморфизма этот предел $\lambda$-п.в. равен среднему значению $\lambda(f)=\int_{\Omega} f d \lambda$.

Скорость сходимости в этой теореме будем измерять скоростью убывания при $n \rightarrow \infty$ для каждого $\varepsilon>0$ величин

$$
\mathrm{P}_{n}^{\varepsilon}=\lambda\left\{\sup _{k \geqslant n}\left|A_{k} f-f^{*}\right| \geqslant \varepsilon\right\}
$$

Оценки для этих величин можно получать с помощью оценок для вероятностей больших уклонений, т.е. для величин $\mathrm{p}_{n}^{\varepsilon}=\lambda\left\{\left|A_{n} f-f^{*}\right| \geqslant \varepsilon\right\}$ (см. [1], [2]). Оказывается, что если для некоторой существенно ограниченной функции $f$ известна не только верхняя оценка, но и точная экспоненциальная асимптотическая формула для вероятностей больших уклонений, то такая же формула верна и для скорости сходимости в теореме Биркгофа (теорема 1 ниже). В качестве примера мы применяем этот результат к транзитивному диффеоморфизму Аносова.

Пусть $f \in L_{\infty}(\Omega), \Delta=\left\|f-f^{*}\right\|_{\infty}$. Будем предполагать, что функция $f \not \equiv f^{*}$ п.в., иначе оценивать нечего: $\mathrm{P}_{n}^{\varepsilon}=0$ для всех $n \in \mathbb{N}$ и $\varepsilon>0$. Для любых действительных $\varepsilon>0$ и $d>$ 1 положим

$$
r=r(\varepsilon, d)=1+\frac{\varepsilon(d-1)}{\Delta} .
$$

Лемма 1. Пусть $f \in L_{\infty}(\Omega)$. Тогда для любых действительных $\varepsilon>0$ u $d>1$

$$
\mathrm{P}_{n}^{d \varepsilon} \leqslant \sum_{k=0}^{\infty} \lambda\left\{\sup _{n r^{k} \leqslant m<n r^{k+1}}\left|A_{m} f-f^{*}\right| \geqslant d \varepsilon\right\} \leqslant \sum_{k=0}^{\infty} \mathrm{p}_{n_{k}}^{\varepsilon} \quad \text { для всех } \quad n \in \mathbb{N},
$$

где $n_{k}$ - минимальное целое число, не менъшее $n r^{k}$.

Лемма 2. Пусть $f \in L_{\infty}(\Omega)$. Если для некоторого $\varepsilon>0$ найдутся такие константы $C(\varepsilon)>0, \gamma(\varepsilon)>0$, что для всех $n>n_{0}(\varepsilon), n_{0}(\varepsilon) \in \mathbb{Z}^{+}$, справедливо неравенство $\mathrm{p}_{n}^{\varepsilon} \leqslant$ $C(\varepsilon) e^{-\gamma(\varepsilon) n}$, mо для всех $n>n_{0}(\varepsilon) u d>1$

$$
\mathrm{P}_{n}^{d \varepsilon} \leqslant C(\varepsilon)\left(1+\frac{\ln (1+1 / \gamma(\varepsilon))}{\ln r(\varepsilon, d)}\right) e^{-\gamma(\varepsilon) n} .
$$

Лемма 1 доказана в [1], [2] для случая $d=2$; для произвольного действительного $d>1$ доказательство аналогично. Лемма 2 доказывается с помощью леммы 1 таким же образом, как в [2; теорема 3 , пункт 3].

Работа выполнена при финансовой поддержке программы "Ведущие научные школы" (грант № НШ-5998.2012.1).

DOI: $10.4213 / \operatorname{mzm} 10430$

(C) И. В. Подвигин, 2014 
Теорема 1. Если для $f \in L_{\infty}(\Omega)$ найдется непрерывная функиия $\gamma_{f}(t): \mathbb{R} \mapsto \mathbb{R}^{+}$такая, что $\gamma_{f}(t)=0$, если и только если $t=0$, и для каждого достаточно малого $\varepsilon>0$

$$
\lim _{n \rightarrow \infty} \frac{1}{n} \ln \mathrm{p}_{n}^{\varepsilon}=-\gamma_{f}(\varepsilon),
$$

то для такой функции $f$ при этих же $\varepsilon>0$

$$
\lim _{n \rightarrow \infty} \frac{1}{n} \ln \mathrm{P}_{n}^{\varepsilon}=-\gamma_{f}(\varepsilon) .
$$

ДокАЗАтельство. Зафиксируем малое $\varepsilon>0$. Для любого $\delta>0$ найдется такое $N_{1}=$ $N_{1}\left(\delta, \gamma_{f}(\varepsilon)\right)$, что для всех $n \geqslant N_{1}$

$$
e^{-\left(\gamma_{f}(\varepsilon)+\delta\right) n} \leqslant \mathbf{p}_{n}^{\varepsilon} \leqslant e^{-\left(\gamma_{f}(\varepsilon)-\delta\right) n} .
$$

Аналогично, для любого $d>1$ (такого, что $d \varepsilon$ оставалось бы малым) и $\delta>0$ найдется такое $N_{2}=N_{2}\left(\delta, \gamma_{f}(d \varepsilon)\right)$, что для всех $n \geqslant N_{2}$

$$
e^{-\left(\gamma_{f}(d \varepsilon)+\delta\right) n} \leqslant \mathbf{p}_{n}^{d \varepsilon} \leqslant e^{-\left(\gamma_{f}(d \varepsilon)-\delta\right) n} .
$$

Используя правую часть неравенства $(1)$ для $\delta<\gamma_{f}(\varepsilon)$ и лемму 2 с константами $C(\varepsilon)=1$, $\gamma(\varepsilon)=\gamma_{f}(\varepsilon)-\delta$ и $n_{0}(\varepsilon)=N_{1}$, получаем для всех $n \geqslant N_{1}$ и $d>1$

$$
\mathrm{P}_{n}^{d \varepsilon} \leqslant\left(1+\frac{\ln \left(1+1 /\left(\gamma_{f}(\varepsilon)-\delta\right)\right)}{\ln r(\varepsilon, d)}\right) e^{-\left(\gamma_{f}(\varepsilon)-\delta\right) n} .
$$

Левая часть неравенства (2) при любых $\delta>0, d>1$ и всех $n \geqslant N_{2}$ дает очевидную оценку

$$
e^{-\left(\gamma_{f}(d \varepsilon)+\delta\right) n} \leqslant \mathbf{p}_{n}^{d \varepsilon} \leqslant \mathbf{P}_{n}^{d \varepsilon} .
$$

Записывая оценки (3) и (4) вместе, для любых $0<\delta<\gamma_{f}(\varepsilon)$ и $d>1$ при всех $n \geqslant N_{3}, N_{3}=$ $N_{3}\left(\delta, \gamma_{f}(\varepsilon), \gamma_{f}(d \varepsilon)\right)=\max \left\{N_{1}, N_{2}\right\}$, получаем неравенства

$$
e^{-\left(\gamma_{f}(d \varepsilon)+\delta\right) n} \leqslant \mathbf{P}_{n}^{d \varepsilon} \leqslant\left(1+\frac{\ln \left(1+1 /\left(\gamma_{f}(\varepsilon)-\delta\right)\right)}{\ln r(\varepsilon, d)}\right) e^{-\left(\gamma_{f}(\varepsilon)-\delta\right) n} .
$$

Полагая

$$
k(\varepsilon, \delta, d)=\ln \left(1+\frac{\ln \left(1+1 /\left(\gamma_{f}(\varepsilon)-\delta\right)\right)}{\ln (1+\varepsilon(d-1) / \Delta)}\right)
$$

и логарифмируя последнее неравенство, получаем

$$
-\gamma_{f}(d \varepsilon)-\delta \leqslant \frac{1}{n} \ln \mathrm{P}_{n}^{d \varepsilon} \leqslant \frac{k(\varepsilon, \delta, d)}{n}-\gamma_{f}(\varepsilon)+\delta .
$$

После замены $\varepsilon d$ на $\varepsilon$ получаем для любых $\varepsilon>0, d>1$ и $0<\delta<\gamma_{f}(\varepsilon / d)$ и при всех $n \geqslant$ $N_{3}\left(\delta, \gamma_{f}(\varepsilon / d), \gamma_{f}(\varepsilon)\right)$

$$
-\gamma_{f}(\varepsilon)-\delta \leqslant \frac{1}{n} \ln \mathrm{P}_{n}^{\varepsilon} \leqslant \frac{k(\varepsilon / d, \delta, d)}{n}-\gamma_{f}\left(\frac{\varepsilon}{d}\right)+\delta .
$$

Воспользуемся теперь непрерывностью $\gamma_{f}$. Для любого $\nu>0$ найдется $\tau=\tau(\nu, \varepsilon)>0$ такое, что $\left|\gamma_{f}(\varepsilon)-\gamma_{f}(\varepsilon / d)\right|<\nu$ при всех $1<d \leqslant \tau$.

Взяв $1<d \leqslant \tau$, неравенство (5) перепишем в виде

$$
-\gamma_{f}(\varepsilon)-\delta \leqslant \frac{1}{n} \ln \mathrm{P}_{n}^{\varepsilon} \leqslant \frac{k(\varepsilon / d, \delta, d)}{n}-\gamma_{f}(\varepsilon)+\nu+\delta .
$$


Отсюда получаем, что для любых $\nu>0$ и $0<\delta<\min _{1 \leqslant d \leqslant \tau} \gamma_{f}(\varepsilon / d)$

$$
\begin{aligned}
& -\delta \leqslant \varlimsup_{n \rightarrow \infty}\left(\frac{1}{n} \ln \mathrm{P}_{n}^{\varepsilon}+\gamma_{f}(\varepsilon)\right) \leqslant \nu+\delta, \\
& -\delta \leqslant \varliminf_{n \rightarrow \infty}\left(\frac{1}{n} \ln \mathrm{P}_{n}^{\varepsilon}+\gamma_{f}(\varepsilon)\right) \leqslant \nu+\delta .
\end{aligned}
$$

Ввиду произвольности $\nu$ и $\delta$ заключаем, что

$$
\lim _{n \rightarrow \infty} \frac{1}{n} \ln \mathrm{P}_{n}^{\varepsilon}=-\gamma_{f}(\varepsilon) .
$$

Ясно, что получившийся предел справедлив для всех $\varepsilon>0$, при которых верно аналогичное соотношение для больших уклонений. Теорема доказана.

Применим теперь полученный результат к транзитивному $C^{2}$-гладкому диффеоморфизму Аносова $T$, определенному на компактном $C^{\infty}$-гладком римановом многообразии $\Omega$. Хорошо известно (см, например, [3]), что для всякого гиббсовского состояния $\lambda=\lambda_{g}$, порожденного гёльдеровской функцией $g$, и для всякой гёльдеровской функции $f$, не когомологичной константе (т.е. не существует такой непрерывной функции $\phi$ и константы $c$, что $f=\phi \circ T-\phi+c$ п.в.), найдутся интервал $[a, b]$ и вещественно аналитическая функция $I(t):[a, b] \mapsto \mathbb{R}^{+}$такие, что

(i) $\lambda(f) \in(a, b)$;

(ii) $I(t)=0$, если и только если $t=\lambda(f)$;

(iii) для любого борелевского множества $J \subset \mathbb{R}$ такого, что

$$
\inf \{I(t): t \in[a, b] \cap \operatorname{cl} J\}=\inf \{I(t): t \in[a, b] \cap \operatorname{int} J\}
$$

будет справедлив принцип больших уклонений

$$
\lim _{n \rightarrow \infty} \frac{1}{n} \ln \lambda\left\{A_{n} f \in J\right\}=-\inf \{I(t): t \in[a, b] \cap J\} .
$$

Обсуждение этого последнего свойства в более общем контексте принципа больших уклонений можно найти, например, в [4; предложение 3.3].

Взяв $J=\{t:|\lambda(f)-t| \geqslant \varepsilon\}$ для $\varepsilon \in\left[0, \varepsilon_{0}\right], \varepsilon_{0}=\min \{\lambda(f)-a, b-\lambda(f)\}$, и применив теорему 1 к указанной динамической системе для гёльдеровской $f$, не когомологичной константе, и непрерывной на интервале $\left[0, \varepsilon_{0}\right]$ функции

$$
\gamma_{f}(t)=\inf \{I(s): s \in[a, \lambda(f)-t] \cup[\lambda(f)+t, b]\},
$$

получим для любого $\varepsilon \in\left(0, \varepsilon_{0}\right)$ точную асимптотику скорости сходимости:

$$
\lim _{n \rightarrow \infty} \frac{1}{n} \ln \mathrm{P}_{n}^{\varepsilon}=-\gamma_{f}(\varepsilon) .
$$

Для гёльдеровской же $f$, когомологичной константе, легко проверяется, что при любом $\varepsilon>0$ будет $\mathrm{P}_{n}^{\varepsilon}=0$ для всех достаточно больших $n$.

В заключение заметим, что аналог теоремы 1 справедлив и для случая непрерывного времени.

\section{СПИСОК ЦИТИРОВАННОЙ ЛИТЕРАТУРЫ}

[1] А. Г. Качуровский, УМН, 51:4 (1996), 73-124. [2] А. Г. Качуровский, И. В. Подвигин, Матем. заметки, 94:4 (2013), 569-577. [3] M. Pollicott, R. Sharp, Comm. Math. Phys., 290:1 (2009), 321-334. [4] R. S. Ellis, Scand. Actuarial J., 1995:1 (1995), 97-142. 